Pesq. Vet. Bras. 38(6):1125-1129, junho 2018

DOI: $10.1590 / 1678-5150-P V B-5410$

\title{
Atividade acaricida de diferentes solventes sobre fêmeas ingurgitadas e larvas de Rhipicephalus microplus ${ }^{1}$
}

\author{
Jaíne S.P. Vasconcellos ${ }^{2}$, Fagner Fernandes ${ }^{2}$, Gustavo C. Cadore ${ }^{2}$, \\ Fabiana R. Ratzlaff², Laís G. Felipetto², Fernanda S.F. Vogel², Sônia Á. Botton ${ }^{2}$ \\ e Luís A. Sangioni ${ }^{2 *}$
}

\begin{abstract}
Vasconcellos J.S.P., Fernandes F., Cadore G.C., Ratzlaff F.R., Felipetto L.G., Vogel F.S.F., Botton S.A. \& Sangioni L.A. 2018. [Acaricidal activity of different solvents on engorged females and larvae of Rhipicephalus microplus.] Atividade acaricida de diferentes solventes sobre fêmeas ingurgitadas e larvas de Rhipicephalus microplus. Pesquisa Veterinária Brasileira 38(6):1125-1129. Laboratório de Doenças Parasitárias, Departamento de Medicina Veterinária Preventiva, Universidade Federal de Santa Maria, Av. Roraima 1000, Camobi, Santa Maria, RS 97105-900, Brazil. E-mail: lasangioni@gmail.com

Cattle ticks cause economic losses to the cattle industry in Brazil. The use of phytotherapics as acaricides has shown efficacy of the control of these parasites. In the present study, the in vitro efficacy of the following drugs was evaluated: dimethyl sulfoxide (DMSO), ethanol, methanol, propylene glycol and polyethylene glycol sorbitan monolaurate at dilutions $100 \%, 20 \%, 10 \%, 5 \%, 1 \%$ e $0.5 \%$. The toxic effect of these solvents on engorged female and larvae of Rhipicephalus microplus was analyzed. The immersion test was used to define the reproductive efficiency of engorged $R$. microplus females and efficiency of the drugs. The immersion test larvae modified syringe was used to analyze of the susceptibility of tick larvae. Propylene glycol and DMSO was used on engorged females and DMSO, ethanol, methanol and propylene glycol, all at concentration of $0.5 \%$, were use on larvae the, showed the lowest mortality rate; this indicate that its use at low concentrations as solvent acaricide in the control of $R$. microplus could be recommended.
\end{abstract}

INDEX-TERMS: Rhipicephalus microplus, solvent, ticks, cattle, Boophilus, toxicoses.

RESUMO.- Os carrapatos causam perdas econômicas à indústria pecuária no Brasil. Dessa forma, o uso de fitoterápicos como acaricidas mostrou eficácia no controle desses parasitas. Neste estudo, foi avaliada a eficácia in vitro dos seguintes fármacos: dimetil sulfóxido (DMSO), etanol, metanol, propilenoglicol e monolaurato de sorbitan polietilenoglicol em diluições de $100 \%, 20 \%, 10 \%, 5 \%, 1 \%$ e $0,5 \%$. 0 efeito tóxico destes solventes em fêmeas e larvas ingurgitadas de Rhipicephalus microplus foi analisado. O teste de biocarrapaticidograma, foi utilizado para definir a eficiência reprodutiva das teleóginas de $R$. microplus e a eficiência dos produtos testados. Para análise da susceptibilidade das larvas foi utilizado o teste por imersão de larvas em seringa

\footnotetext{
${ }^{1}$ Recebido em 5 de maio de 2017.

Aceito para publicação em 19 de maio de 2017.

${ }^{2}$ Laboratório de Doenças Parasitárias, Departamento de Medicina Veterinária Preventiva, Centro de Ciências Rurais (CCR), Universidade Federal de Santa Maria UFSM, Santa Maria, Camobi, RS 97105-900, Brasil. *Autor para correspondência: lasangioni@gmail.com
}

modificado. O uso de propilenoglicol e DMSO em fêmeas ingurgitadas e o uso de DMSO, etanol, metanol e propilenoglicol em larvas, todos à concentração de $0,5 \%$, apresentaram uma menor taxa de mortalidade, podendo ter seu uso indicado em baixas concentrações como solventes de acaricidas utilizados no controle do R. microplus.

TERMOS DE INDEXAÇÃO: Rhipicephalus microplus, solvente, ixodideo, bovinos, Boophilus, toxicoses.

\section{INTRODUÇÃO}

O Rhipicephalus microplus, tem distribuição cosmopolita, mas ocorre principalmente em áreas tropicais e subtropicais, causando prejuízos econômicos pra bovinocultura (Holdsworth et al. 2006). Devido ao uso indiscriminado de acaricidas comerciais, os carrapatos vêm desenvolvendo mecanismos de resistência aos produtos químicos, relatados em diversos países (Fernández-Salas et al. 2012, Sharma et al. 2012b, 
Machado et al. 2014, Puerta et al. 2015). Carrapaticidas contendo substâncias vegetais são formas alternativas ao controle químico dos carrapatos, que usualmente são utilizados no controle integrado desta parasitose (Zaman et al. 2012). Diversos solventes vêm sendo empregados nas formulações de carrapaticidas ou em experimentos com extratos vegetais (Gonçalves et al. 2007, Monteiro et al. 2012, Sharma et al. 2012a, Valente et al. 2014). Estes produtos possuem o propósito de solubilizar o princípio ativo, permitindo uma distribuição homogênea por toda a cutícula do artrópode para ampliar sua ação (Chagas et al. 2003).

Os extratos vegetais geralmente apresentam baixa solubilidade em água, sendo assim necessário o uso de solventes orgânicos para extração de seus compostos ativos, que promovem a dissolução de seus constituintes armazenados nos diferentes compartimentos da planta, com sua posterior lixiviação para o meio exterior (Hall et al. 1990). A qualidade final de um extrato depende diretamente da seleção do solvente utilizado para sua obtenção (Raaman 2006), sendo assim importante que estes produtos não apresentem efeitos sobre os carrapatos (Ravindran et al. 2011a). Estudos que tratam dos efeitos acaricidas sobre larvas de $R$. microplus não são muito comuns e a maioria dos testes in vitro visa à detecção da ação de mortalidade sobre as fêmeas ingurgitadas do carrapato. Desta maneira, o objetivo deste estudo foi avaliar a ação acaricida de cinco solventes em diferentes concentrações, sobre as fases de teleóginas e larvas do R. microplus, para identificar qual a concentração e o solvente mais indicado para a preparação e obtenção de extratos vegetais para o controle do carrapato.

\section{MATERIAL E MÉTODOS}

Para a realização deste estudo foram utilizados os seguintes solventes: dimetil sulfóxido (DMSO), etanol, metanol, propilenoglicol e monolaurato de sorbitan polietilenoglicol (tween $20^{\circledR}$, Vetec Química Fina Ltda, Duque de Caxias/RJ, Brasil). Para a realização das diluições foi utilizado água ultra-pura, sendo considerada a pureza analítica de cada produto, para obtenção das concentrações de 100\%, 20\%, $10 \%, 5 \%, 1 \%$ e $0,5 \%$.

Teste de imersão de teleóginas. Para avaliar a sensibilidade das fêmeas ingurgitadas de Rhipicephalus microplus, foram coletadas aproximadamente 1000 teleóginas de bovinos, em uma única propriedade rural onde os animais não recebiam produtos acaricidas por no mínimo 30 dias, localizada no estado do Rio Grande do Sul. Foi realizado o teste in vitro de imersão de teleóginas (biocarrapaticidograma) conforme Drummond et al. (1973) e Camillo et al. (2009), sendo estes executados em triplicatas. Foram formados 32 diferentes grupos, constituídos pelos seguintes solventes em diferentes diluições: DMSO, etanol, metanol, propilenoglicol e monolaurato de sorbitan polietilenoglicol (diluições de 100\%, 20\%, 10\%, 5\%, 1\% e 0,5\%); controle negativo (água destilada), controle positivo (clorpirifós $30 \%$, cipermetrina $15 \%$ e fenthion $15 \%$ ). Para seleção do produto utilizado como controle positivo, foi realizado anteriormente um teste de eficácia de acaricidas, com este apresentando uma eficiência de $100 \%$. A eficiência dos solventes foi determinada baseado na fórmula sugerida por Drummond et al. (1973):

Eficiência Reprodutiva (ER) = (peso dos ovos X \% de eclosão dos ovos X 20000)/peso das teleóginas

Eficiência do Solvente $=($ ER do grupo controle - ER do grupo tratado X 100)/ER do grupo controle
Teste de imersão de larvas (TIL). Para avaliar a sensibilidade das larvas de R. microplus, 500 teleóginas foram coletadas. Após pesagem, os carrapatos foram selecionados em grupos homogêneos e fixados em placas de Petri, mantidas em estufa de demanda biológica de oxigênio (DBO), com temperatura de $27^{\circ} \mathrm{C}$ e umidade de $90 \%$, por 14 dias para oviposição. Os ovos das teleóginas foram coletados para a realização do teste de imersão de larvas (TIL) conforme Sindhu et al. (2012). Os ovos foram pesados e colocados em seringas de $3 \mathrm{ml}$, com a extremidade anterior seccionada, coberta com tecido de malha ultrafina e fixada por elástico ortodôntico. Em cada seringa foram colocados $10 \mathrm{mg}$ de ovos (aproximadamente 200 ovos), sendo posteriormente acondicionados em estufa DBO para a incubação e eclosão dos ovos. As seringas que não apresentaram eclosão de $100 \%$ dos ovos foram descartadas. Trinta e dois grupos foram constituídos pelos seguintes solventes em diferentes diluições: DMSO, etanol, metanol, propilenoglicol e monolaurato de sorbitan polietilenoglicol (diluições de 100\%, 20\%, 10\%, 5\%, 1\% e 0,5\%); controle negativo (água destilada); controle positivo (clorpirifós $30 \%$, cipermetrina $15 \%$ e fenthion $15 \%$ ). Juntamente às larvas eclodidas em cada seringa, foi adicionado $3 \mathrm{ml}$ de cada solução a ser testada por 30 segundos, com posterior remoção do produto. Para avaliação da mortalidade das larvas, cada seringa foi observada em microscópio esterioscópico em 24 horas e 6 dias após os banhos de imersão (Sindhu et al. 2012). Larvas que apresentavam movimentos nas patas ou se deslocavam, foram consideradas vivas. A mortalidade das larvas foi expressa em porcentagens, sendo consideradas as médias de mortalidade encontradas para cada solvente testado nas suas diferentes concentrações. Para o cálculo da mortalidade das larvas foi considerada a seguinte fórmula:

Mortalidade $=($ larvas mortas X 100)/total de larvas

Análise estatística. Todos os dados para avaliação estatística foram realizados através do programa SAS (SAS Institute Inc., Cary, NC). A significância das eficácias dos solventes e das diferentes concentrações foram analisadas através do teste de Kruskal-Wallis, com uma confiança de 95\%.

\section{RESULTADOS E DISCUSSÃO}

Neste estudo 5 solventes foram testados in vitro, em diferentes concentrações, com o objetivo de avaliar seus efeitos acaricidas sobre fêmeas adultas e larvas do Rhipicephalus microplus. Os resultados das médias de eficácia após o teste de imersão das fêmeas ingurgitadas dos carrapatos, nos solventes testados em diferentes concentrações, estão demonstrados na Quadro 1. Os controles positivos e negativos apresentaram eficácias de $100 \%$ e $0 \%$ respectivamente (dados não mostrados). 0 uso de dimetil sulfóxido (DMSO), propilenoglicol e monolaurato de sorbitan polietilenoglicol utilizados na concentração de $100 \%$, inibiram totalmente a postura das fêmeas ingurgitadas, demonstrando uma eficácia de $100 \%$, ou seja, estes solventes nesta concentração apresentaram toxicidade total para as teleóginas (Quadro 1). Somente foram observadas diferenças estatísticas significativas entre os diferentes solventes na concentração de $0,5 \%$, onde apenas o DMSO e o propilenoglicol, apresentaram uma menor toxicidade que os demais (Quadro 1). Para as diferentes concentrações aplicadas dentro de um mesmo solvente, foi verificado que apenas o DMSO nas concentrações de $5 \%, 1 \%$ e $0,5 \%$ e o propilenoglicol nas concentrações de $10 \%, 5 \%, 1 \%$ e $0,5 \%$ apresentaram menor toxicidade significativa em comparação com os demais produtos (Quadro 1). 
Os resultados da avaliação das médias de mortalidade de larvas de R. microplus em 24 horas e 6 dias após imersão nos solventes testados nas diferentes concentrações estão demonstrados na Quadro 2. Os controles positivos e negativos das larvas apresentaram mortalidade de $100 \%$ e $0 \%$ respectivamente (dados não mostrados). 0 uso de DMSO, metanol, propilenoglicol e monolaurato de sorbitan polietilenoglicol, na concentração de $100 \%$ em 24 horas após imersão e o uso DMSO a 20\% em 6 dias após imersão, apresentaram toxicidade máxima com mortalidade total das larvas (Quadro 2). 0 DMSO é considerado um excelente permeabilizador de membranas, permitindo a penetração de produtos a serem testados de maneira eficiente (Chagas et al. 2003). A ação tóxica que o DMSO apresenta, já havia sido verificada em pesquisa anterior, porém em concentrações mais elevadas (Chagas et al. 2003). Concentrações de até $10 \%$ podem ser utilizadas com baixa mortalidade, porém concentrações acima de $20 \%$ demonstram altas taxas de letalidade em fêmeas adultas (Gonçalves et al. 2007, Sharma et al. 2012a). Neste estudo, em larvas do carrapato na concentração de $0,5 \%$ do DMSO, resultou em uma média de mortalidade de $5,6 \%$ e $7,3 \%$ no período de 24 horas e 6 dias após imersão, respectivamente, indicando uma baixa toxicidade deste solvente nestas concentrações (Quadro 2).
De maneira semelhante, Resende et al. (2012) observaram que o DMSO a 1\%, em teste de imersão em larvas de carrapatos dos gêneros Amblyomma cajennense e Dermacentor nitens, mostrou baixa toxicidade com índices de $4 \%$ de mortalidade. Desta maneira, o DMSO pode causar altas taxas de mortalidade, em concentrações mais elevadas, tendo seu uso contra indicado como solvente de extratos vegetais que serão utilizados no controle do R. microplus.

0 etanol e o metanol apresentaram um alto índice de mortalidade no seu uso tanto em fêmeas adultas (Quadro 1), quanto em larvas do carrapato (Quadro 2), porém o uso de etanol a $0,5 \%$ demonstrou pequena taxa de mortalidade ( $5 \%$ em 24 horas e 6,6\% em 6 dias, após imersão). Conforme Gonçalves et al. (2007), empregando o etanol no teste de imersão em fêmeas ingurgitadas de R. microplus, verificaram que este solvente apresentou máxima ação tóxica. Para o metanol, resultados semelhantes também indicam presença de toxicidade em teleóginas, não sendo indicados como solventes adequados para testes acaricidas nesta espécie de carrapato (Chagas et al. 2003, Gonçalves et al. 2007). No entanto Sharma et al. (2012a), consideraram que concentrações de etanol menores de $25 \%$ e de metanol menores que $50 \%$ como seguras em fêmeas ingurgitadas submetidas à imersão. Nas larvas, estudos anteriores demonstraram baixa toxidez

Quadro 1. Média de eficácia (\%) dos solventes, após imersão de fêmeas ingurgitadas de Rhipicephalus microplus em dimetil sulfóxido (DMSO), etanol, metanol, propilenoglicol e monolaurato de sorbitan polietilenoglicol, em diferentes concentrações

\begin{tabular}{|c|c|c|c|c|c|c|}
\hline \multirow[t]{2}{*}{ Solventes } & \multicolumn{6}{|c|}{$\begin{array}{c}\text { Concentração dos solventes } \\
(\%)\end{array}$} \\
\hline & 100 & 20 & 10 & 5 & 1 & 0,5 \\
\hline DMSO & $100^{\mathrm{aA}}$ & $78,5^{\mathrm{aAB}}$ & $72^{\mathrm{aAB}}$ & $27^{\mathrm{aB}}$ & $29,5^{\mathrm{aB}}$ & $18^{\mathrm{aB}}$ \\
\hline Etanol & $98^{\mathrm{aA}}$ & $69,5^{\mathrm{aA}}$ & $73^{\mathrm{aA}}$ & $50^{\mathrm{aA}}$ & $50^{\mathrm{aA}}$ & $45,5^{\mathrm{abA}}$ \\
\hline Metanol & $97,5^{\mathrm{aA}}$ & $79^{\mathrm{aA}}$ & $76^{\mathrm{aA}}$ & $72,5^{\mathrm{aA}}$ & $71^{\mathrm{aA}}$ & $65^{\mathrm{bA}}$ \\
\hline Propilenoglicol & $100^{\mathrm{aA}}$ & $93,5^{\mathrm{aA}}$ & $31^{\mathrm{aB}}$ & $26,5^{\mathrm{aB}}$ & $20^{\mathrm{aB}}$ & $7,5^{\mathrm{aB}}$ \\
\hline Monolaurato de sorbitan polietilenoglicol & $100^{\mathrm{aA}}$ & $90,5^{\mathrm{aA}}$ & $84^{\mathrm{aA}}$ & $61,5^{\mathrm{aA}}$ & $49,5^{\mathrm{aA}}$ & $57^{\mathrm{abA}}$ \\
\hline
\end{tabular}

Letras minúsculas distintas se referem à diferença estatística entre a mesma concentração de diferentes solventes, avaliados de maneira isolada, através do teste de Kruskal-Wallis $(\mathrm{p}<0,05)$. Letras maiúsculas distintas se referem à diferença estatística entre as diferentes concentrações de um mesmo solvente, avaliados de maneira isolada, através do teste de Kruskal-Wallis $(\mathrm{p}<0,05)$.

Quadro 2. Média de mortalidade de larvas de Rhipicephalus microplus 24 horas e 6 dias após imersão em dimetil sulfóxido (DMSO), etanol, metanol, propilenoglicol e monolaurato de sorbitan polietilenoglicol, em diferentes concentrações

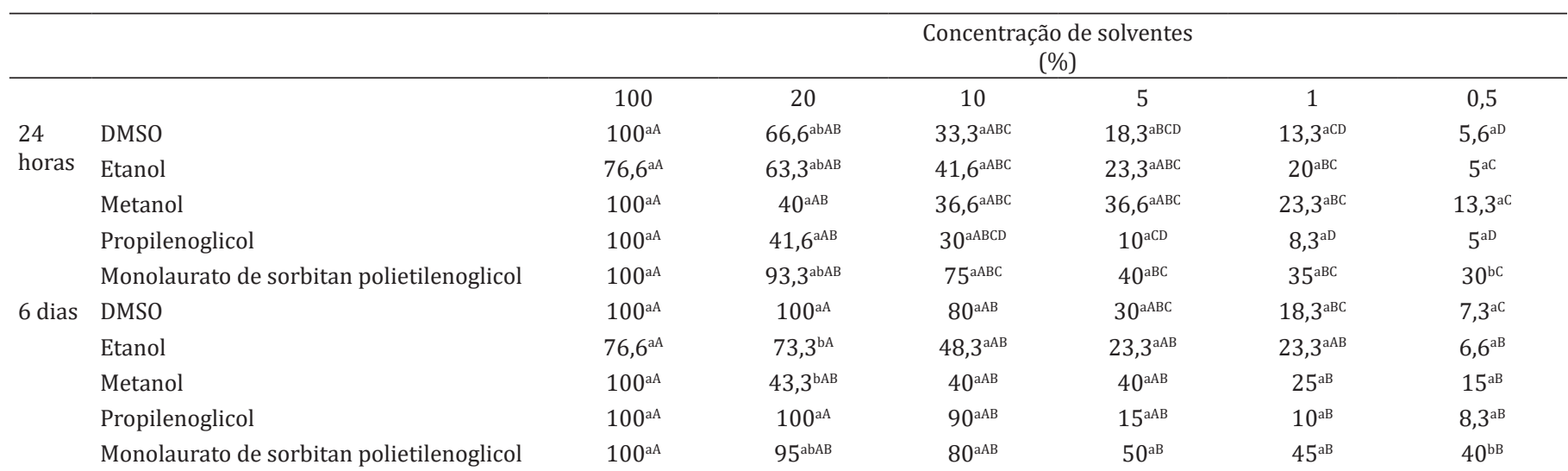

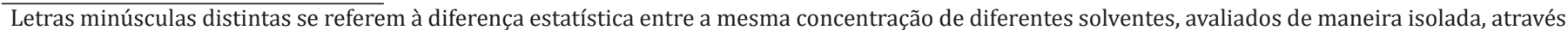

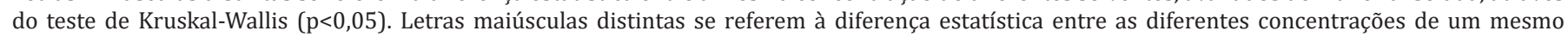
solvente, avaliados de maneira isolada, através do teste de Kruskal-Wallis $(\mathrm{p}<0,05)$. 
do etanol e do metanol, mesmo em elevadas concentrações (Chagas et al. 2003, Gonçalves et al. 2007). Assim, vale ressaltar que as diferenças de susceptibilidade aos solventes empregados podem ser atribuídas a diversos fatores, que vão desde a composição da cutícula do artrópode, diferentes técnicas de imersão utilizadas e diferenças entre as cepas carrapatos (Resende et al. 2012).

0 teste empregando o solvente propilenoglicol, apresentou alto índice de eficácia em teleóginas, nas concentrações de $100 \%$ a 1\% (Quadro 1). No teste de imersão das larvas, apresentou baixa toxicidade apenas na concentração de $0,5 \%$ no período de 24 horas após imersão (Quadro 2). 0 propilenoglicol é um solvente viscoso, utilizado em uma grande variedade de formulações farmacêuticas, sendo empregado na preparação de extratos vegetais (Anchisi et al. 2001, Jaenson et al. 2006, Pohlit et al. 2011). Os relatos científicos avaliando o propilenoglicol em produtos acaricidas são bastante escassos, portanto este solvente combinado com fungicidas melhorou significativamente a eficiência de produtos testados in vitro sobre o fungo Bipolaris sorokiniana em sementes de cevada (Barba et al. 2003).

0 teste utilizando monolaurato de sorbitan polietilenoglicol foi tóxico em todas suas concentrações nos testes realizados (Quadros 1 e 2). Concentrações acima de 8\% do monolaurato de sorbitan polietilenoglicol, já apresentaram altas taxas de mortalidade em fêmeas adultas, porém não foram observadas taxas de mortalidade nem impactos negativos na realização de ovipostura, quando este solvente foi utilizado em concentrações menores de 6\% (Sharma et al. 2012a). Em estudo anterior utilizando o monolaurato de sorbitan polietilenoglicol a 1\%, apresentou baixo efeito tóxico contra carrapatos adultos de Rhipicephalus annulatus, no entanto esta concentração resultou em efeito inibitório sobre a eclosão dos ovos (Ravindran et al. 2011b). Este solvente apresenta característica lipofílica, podendo remover a camada epicuticular dos carrapatos e desta maneira favorecer a absorção de princípios ativos, podendo resultar em mortalidade dos ixodideos (Odhiambo 1982). Neste estudo, foi verificado que em nenhuma das concentrações testadas para as larvas a mortalidade média foi inferior a 30\% (Quadro 2), portanto sendo considerado um solvente de alta toxicidade.

Os resultados encontrados demonstraram que as teleóginas apresentaram maiores taxas de mortalidade quando comparado as larvas. Essa diferença pode ser relativa à composição da cutícula entre estas duas fases de desenvolvimento dos carrapatos (Chagas et al. 2003). A camada cuticular externa dos carrapatos adultos é composta principalmente por ceras e proteínas, podendo ser observada uma maior quantidade de lipídeos na cutícula do R. microplus a partir da ecdise da ninfa para a fase adulta (Balashov 1972), portanto as larvas não possuem esta camada de cera o que pode ter permitido uma maior ação dos solventes. Essa composição da cutícula dos artrópodes também pode justificar a ação acaricida observada nos testes com os solventes DMSO, propilenoglicol e monolaurato de sorbitan polietilenoglicol em altas concentrações. Sendo assim, quanto maior a lipossolubilidade do produto, maior será a capacidade dos princípios ativos penetrarem na cutícula do carrapato (Odhiambo 1982).

\section{CONCLUSÕES}

O propilenoglicol a $0,5 \%$ apresentou o menor índice de eficácia em teleóginas.

Na avaliação das larvas, o dimetil sulfóxido (DMSO), etanol e o propilenoglicol, utilizados na concentração de $0,5 \%$ foram os solventes que apresentaram menores efeitos tóxicos.

As fêmeas ingurgitadas, quando comparadas às larvas, demonstraram ser mais sensíveis aos solventes utilizados.

Pela ação conjunta, tanto em fêmeas adultas quanto em larvas de Rhipicephalus microplus, o propilenoglicol e DMSO a $0,5 \%$ foram os solventes que demonstraram as menores taxas de toxicidade, demonstrando pouco efeito acaricida, podendo ser indicados como possíveis solventes em soluções carrapaticidas.

Agradecimentos.- À Coordenação de Aperfeiçoamento de Pessoal de Nível Superior (CAPES) pelo importante auxílio através de bolsa.

\section{REFERÊNCIAS}

Anchisi C., Maccioni A.M., Sinico C. \& Valenti D. 2001. Stability studies of new cosmetic formulations with vegetable extracts as functional agents. Il Farmaco 56(5/7):427-431. http://dx.doi.org/10.1016/S0014-827X(01)01055-2. PMid:11482771.

Balashov I.U.S. 1972. A translation of bloodsucking ticks (Ixodidea): vectors of diseases of man and animals. Misc. Publ. Entomol. Soc. Am. 8(5):159-376.

Barba J.T., Reis E.M. \& Forcelini C.A. 2003. Efeito dos solventes orgânicos usados como veículos de fungicidas no controle in vitro e in vivo da incidência e da transmissão de Bipolaris sorokiniana em sementes de cevada. Fitopatol. Bras. 28(2):136-142. http://dx.doi.org/10.1590/S0100-41582003000200003.

Camillo G., Vogel F.S.F., Sangioni S.A., Cadore G.C. \& Ferrari R. 2009. Eficiência in vitro de acaricidas sobre carrapatos de bovinos no Estado do Rio Grande do Sul, Brasil. Ciência Rural 39(2):490-495. http://dx.doi.org/10.1590/ S0103-84782008005000082.

Chagas A.C.S., Leite R.C., Furlong J., Prates H.T. \& Passos W.M. 2003. Sensibilidade do carrapato Boophilus microplus a solventes. Ciência Rural 33(1):109-114. http://dx.doi.org/10.1590/S0103-84782003000100017.

Drummond R.O., Ernst S.E., Trevino J.L., Gladney W.J. \& Graham O.H. 1973. Boophilus annulatus and B. microplus: laboratory tests of insecticides. J. Econ. Entomol. 66(1):130-133. http://dx.doi.org/10.1093/jee/66.1.130. PMid:4690254.

Fernández-Salas A., Rodríguez-Vivas R.I. \& Alonso-Díaz M.A. 2012. First report of a Rhipicephalus microplus tick population multi-resistant to acaricides and ivermectin in the Mexican tropics. Vet. Parasitol. 183(3/4):338-342. http://dx.doi.org/10.1016/j.vetpar.2011.07.028. PMid:21824728.

Gonçalves K., Toigo E., Ascoli B., von Poser G. \& Ribeiro V.L. 2007. Effects of solvents and surfactant agents on the female and larvae of cattle tick Boophilus microplus. Parasitol. Res. 100(6):1267-1270. http://dx.doi. org/10.1007/s00436-006-0418-2. PMid:17237954.

Hall D.W., Sandrin J.A. \& McBride R.E. 1990. An overview of solvent extraction treatment technologies. Environ. Prog. Sustainable Energy 9(2):98-105.

Holdsworth P.A., Kemp D., Green P., Peter R.J., De Bruin C., Jonsson N.N., Letonja T., Rehbein S. \& Vercruysse J. 2006. World Association for the Advancement of Veterinary Parasitology (W.A.A.V.P.), guidelines for evaluating the efficacy of acaricides against ticks (Ixodidae) on ruminants. Vet. Parasitol. 136(1):29-43. https://doi.org/10.1016/j.vetpar.2005.11.011.

Jaenson T.G.T., Garboui S. \& Palsson K. 2006. Repellency of oils of lemon eucalyptus, geranium, and lavender and the mosquito repellent MyggA natural to Ixodes ricinus (Acari: Ixodidae) in the laboratory and field. J. Med. Entomol. 43(4):731-736. http://dx.doi.org/10.1093/jmedent/43.4.731. PMid:16892632. 
Machado F.A., Pivoto F.L., Ferreira M.S.T., Gregorio F.V., Vogel F.S.F. \& Sangioni L.A. 2014. Rhipicephalus (Boophilus) microplus in the western-central region of Rio Grande do Sul, Brazil: multiresistant tick. Revta Bras. Parasitol. Vet. 23(3):337-342. http://dx.doi.org/10.1590/S1984-29612014063.

Monteiro C.M., Maturano R., Daemon E., Catunda-Junior F.E., Calmon F., Senra T.S., Faza A. \& Carvalho M.G. 2012. Acaricidal activity of eugenol on Rhipicephalus microplus (Acari: Ixodidae) and Dermacentor nitens (Acari: Ixodidae) larvae. Parasitol. Res. 111(3):1295-1300. http://dx.doi. org/10.1007/s00436-012-2964-0. PMid:22622689.

Odhiambo T.R. 1982. Current Themes in Tropical Science: physiology of ticks. Pergamon, Oxford. 508p.

Pohlit A.M., Rezende A.R., Lopes Baldin E.R., Lopes N.P. \& Andrade Neto V.F. 2011. Plant extracts, isolated phytochemicals, and plant-derived agents which are lethal to arthropod vectors of human tropical diseases, a review. Planta Med. 77(6):618-630. http://dx.doi.org/10.1055/s-0030-1270949. PMid:21432748.

Puerta J.M., Chaparro J.J., Lopez-Arias A., Arroyave S.A. \& Villar D. 2015. Loss of in vitro efficacy of topical commercial Acaricides on Rhipicephalus microplus (Ixodida: Ixodidae) from Antioquian farms, Colombia. J. Med. Entomol. 52(6):1309-1314. https://doi.org/10.1093/jme/tjv129.

Raaman N. 2006. Phytochemical Methods. Nem India Publishing Agency, New Delhi. 296p.

Ravindran R., Juliet S., Kumar K.G., Sunil A.R., Nair S.N., Amithamol K.K., Rawat A.K. \& Ghosh S. 2011a. Toxic effects of various solvents against Rhipicephalus (Boophilus) annulatus. Ticks Tick Borne Dis. 2(3):160-162. http://dx.doi. org/10.1016/j.ttbdis.2011.04.001. PMid:21890070.

Ravindran R., Juliet S., Gopalan A.K.K., Kavalimakkil A.K., Ramankutty S.A., Nair S.N., Narayanan P.M. \& Ghosh S. 2011b. Toxicity of DMSO, Triton X
100 and tween 20 against Rhipicephalus (Boophilus) annulatus. J. Parasit. Dis. 35(2):237-239. http://dx.doi.org/10.1007/s12639-011-0054-3. PMid:23024514.

Resende J.D.S.A., Daemon E., Monteiro C.M., Maturano R., Prata M.C. \& Rodrigues A.F.F. 2012. Toxicity of solvents and surfactants to Amblyomma cajennense (Fabricius, 1787) (Acari: Ixodidae) and Dermacentor nitens (Neumann, 1897) (Acari: Ixodidae) larvae. Exp. Parasitol. 131(2):139-142. http:// dx.doi.org/10.1016/j.exppara.2012.03.002. PMid:22459626.

Sharma A.K., Kumar S., Tiwari S.S. \& Ghosh S. 2012a. Comparative acaricidal properties of different solvents and surfactants on Rhipicephalus (Boophilus) microplus (Acari: Ixodidae). Indian J. Anim. Sci. 82(2):154-158.

Sharma A.K., Kumar R., Kumar S., Nagar G., Singh N.K., Rawat S.S., Dhakad M.L., Rawat A.K., Ray D.D. \& Ghosh S. 2012b. Deltametrin and cypermethrin resistance status of Rhipicephalus (Boophilus) microplus collected from six agro-climatic regions of India. Vet. Parasitol. 188(3/4):337-345. http:// dx.doi.org/10.1016/j.vetpar.2012.03.050. PMid:22541585.

Sindhu Z.-D., Jonsson N.N. \& Igbal Z. 2012. Syringe test (modified larval immersion test): a new bioassay for testing acaricidal activity of plant extracts against Rhipicephalus microplus. Vet. Parasitol. 188(3/4):362 367. http://dx.doi.org/10.1016/j.vetpar.2012.03.021. PMid:22516644.

Valente P.P., Amorim J.M., Castilho R.O., Leite R.C. \& Ribeiro M.F. 2014. In vitro acaricidal efficacy of plant extracts from Brazilian flora and isolated substances against Rhipicephalus microplus (Acari: Ixodidae). Parasitol. Res. 113(1):417-423. http://dx.doi.org/10.1007/s00436-013-3670-2. PMid:24221889.

Zaman M.A., Iqbal Z., Abbas R.Z., Khan M.N., Muhammad G., Younus M. \& Ahmed S. 2012. In vitro and in vivo acaricidal activity of a herbal extract. Vet Parasitol.186(3/4):431-436. https://doi.org/10.1016/j.vetpar.2011.11.018. 